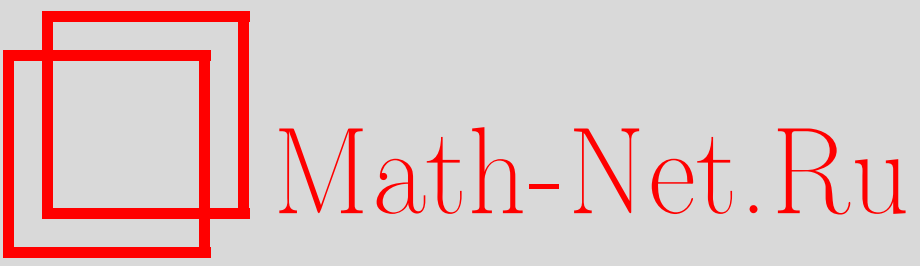

Ю. Н. Обухов, Калибровочные поля и геометрия пространства-времени, ТМФ, 1998, том 117, номер 2, 249-262

DOI: https://doi.org/10.4213/tmf930

Использование Общероссийского математического портала Math-Net.Ru подразумевает, что вы прочитали и согласны с пользовательским соглашением

http://www.mathnet.ru/rus/agreement

Параметры загрузки:

IP : 34.229 .45 .116

26 апреля 2023 г., 13:23:42 


\author{
ТЕОРЕТИЧЕСКАЯ \\ И МАТЕМАТИЧЕСКАЯ \\ ФИЗИКА \\ Том 117, № 2 \\ ноябрь, 1998
}

(C) 1998 г.

Ю.Н. Обухов*

\title{
КАЛИБРОВОЧНЫЕ ПОЛЯ И ГЕОМЕТРИЯ ПРОСТРАНСТВА-ВРЕМЕНИ
}

Рассматриваются отображения между $S U(2)$-калибровочной теорией Янга-Миллса и калибровочной теорией гравитации. Глобальные аспекты обсуждаются в рамках формализма расслоений, и дается подробное описание трех локальных конструкций (в зависимости от существования фиксированной фоновой метрики).

\section{1. ВВЕДЕНИЕ}

Понятия калибровочной симметрии и калибровочного поля лежат в основе современных представлений о природе физических взаимодействий. Математические формулировки выявляют важную роль расслоений и их геометрических и топологических свойств [1]. Сушествуют два типа калибровочных полей, которые отвечают "внутренним" и “внешним" (или пространственно-временным) симметриям. Пространственно-временные симметрии и "внешние" калибровочные поля связаны с гравитацией. Эйнштейновская общая теория относительности, которая описывает гравитационные явления на больших расстояниях, хорошо понятна в рамках калибровочного подхода на основе группы диффеоморфизмов пространства-времени и так называемого предела телепараллелизма для калибровочной группы Пуанкаре. На малых расстояниях можно естественным образом ожидать расширения теории до обшей пуанкаре- (и/или конформной и аффинной) калибровочной теории гравитации [2]. "Внутренние" калибровочные теории электрослабых и сильных взаимодействий (стандартная модель и квантовая хромодинамика) успешно описывают явления в физике частиц высоких энергий [3]. Эти модели обычно основаны на унитарных группах $U(1), S U(N), N=2,3, \ldots$, хотя ряд схем "великого объединения" иногда строится на основе ортогональных или исключительных групп. Неоднократно предпринимались попытки связать "внешние" и "внутренние" калибровочные теории. Некоторый прогресс был недавно достигнут в рамках супергравитации и теории суперструн. С геометрической точки зрения связи между “внешней” и “внутренней” геометриями строятся в этих моделях с помошью определенных вложений их в более сложную структуру, такую как супергеометрия и бесконечномерная геометрия протяженных объектов.

В то же время можно установить формальные связи между “внешней" и "внутренней" геометриями более непосредственным образом. В качестве первого примера отметим

${ }^{*}$ Институт теоретической физики, Университет Кельна, Кельн, ФРГ 
подход Аштекара в квантовой гравитации [4]. Общая теория относительности оперирует с геометрией четырехмерного многообразия, описываемой посредством (псевдо) римановой метрической структуры, причем в каноническом подходе центральную роль играет 3-геометрия пространства. В подходе Аштекара вводится фундаментальная структура связности на основе калибровочной $S O(3) \cong S U(2)$-группы таким образом, что "внешняя" метрическая 3-геометрия возникает как "эффективная" структура из "внутренней" калибровочной структуры янг-миллсовского типа со специальным функционалом Гамильтона. Замечательным образом этот подход допускает ясную формулировку в рамках пуанкаре-калибровочной теории [5]. Более того, доказательство положительности энергии значительно упрошается в новых полевых переменных [6]. Таким образом, подход Аштекара можно рассматривать как реализацию отображений некоторой "внутренней" калибровочной теории во "внешнюю" калибровочную теорию.

Сушествуют примеры отображений в "противоположном направлении". Калибровочная теория Янга-Миллса для группы $S U(N)(N=2,3, \ldots)$ лежит в основе квантовой хромодинамики (КХД), которая удовлетворительно описывает адронные явления при больших значениях переданного импульса [7]. Асимптотическая свобода позволяет использовать теорию возмушений. В работе [7] отмечалось: "Однако наиболее важные явления в сильных взаимодействиях нельзя описать пертурбативными методами, и в настояшее время "непертурбативная КХД" является для нас величайшим вызовом". А дроны рассматриваются как элементарные возбуждения вакуума КХ Д, и полное понимание природы адронов сушественным образом связано с проблемой вакуума (основного состояния) в модели Янга-Миллса. Проблема глюонной материи (глюболов, описываемых посредством одних только полей Янга-Миллса) также связана со структурой вакуума КХД. Исследуя последнюю в явно калибровочно-инвариантном формализме, авторы работ [8] предложили подход, в котором динамика полей Янга-Миллса в $(2+1)$ и $(3+1)$ измерениях формулируется в терминах “эффективной” метрической римановой геометрии на 3 -пространстве. Независимо Лунев в серии статей [9] показал возможность "индуцирования" геометрии Римана-Картана в $S U(2)$-калибровочной теории Янга-Миллса. В рамках $S U(3)$-модели КХД в работе [10] построена калибровочная теория гравитации для группы диффеоморфизмов. Геометрическое обсуждение этих построений было дано в статье [11]. Наконец, недавно еще один альтернативньй подход был предложен в [12].

В данной работе обсуждаются локальные и глобальные аспекты отображений меж-

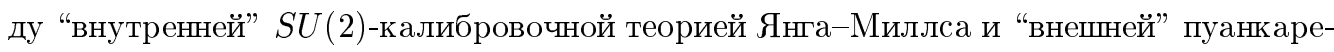
калибровочной теорией.

Эта статья посвяшена памяти Федора Александровича Лунева, с которым мы много лет взаимодействовали в научном и личном плане и который внес весомый вклад в развитие проблемы.

\section{2. РАССЛОЕНИЯ И СВЯЗНОСТИ}

Пусть $M$ - гладкое многообразие, $P(M, G)$ и $P^{\prime}\left(M, G^{\prime}\right)$ суть главные расслоения над $M$ со структурными группами $G$ и $G^{\prime}$, соответственно. Пусть $f^{\prime}: G^{\prime} \rightarrow G$ - гомоморфизм структурных групп. Отображение $f: P^{\prime} \rightarrow P$ называется гомоморфизмом главных расслоений, если $f\left(u^{\prime} g^{\prime}\right)=f\left(u^{\prime}\right) f^{\prime}\left(g^{\prime}\right)$ для всех точек $u^{\prime} \in P^{\prime}$ и групповых эле- 
ментов $g^{\prime} \in G^{\prime}$. Связности в $P^{\prime}$ и $P$ соотносятся следующим образом [13]: если гомоморфизм расслоений $f: P^{\prime}\left(M, G^{\prime}\right) \rightarrow P(M, G)$ таков, что индуцированное им отображение $f: M \rightarrow M$ является диффеоморфизмом, то для любой связности $\Gamma^{\prime}$ в $P^{\prime}$ с некоторой 1-формой связности $\omega^{\prime}$ сушествует единственная связность $\Gamma$ в $P$ такая, что соответствуюшая 1-форма связности $\omega$ удовлетворяет условию

$$
f^{\prime}\left(\omega^{\prime}\left(X^{\prime}\right)\right)=\omega\left(f_{*} X^{\prime}\right)
$$

для любых векторов $X^{\prime} \in T_{u^{\prime}} P^{\prime}, u^{\prime} \in P^{\prime}$. Здесь, как обычно, $f_{*}$ обозначает дифференциал отображения $f$, в то время как $f^{\prime}$ в левой части - гомоморфизм соответствующих алгебр Ли $\mathcal{G}^{\prime} \rightarrow \mathcal{G}$, индуцированный отображением групп $f^{\prime}: G^{\prime} \rightarrow G$. Гомоморфизм $f$ отображает $\Gamma^{\prime}$-горизонтальные подпространства $P^{\prime}$ в горизонтальные подпространства $Г$ в $P$.

Далее, положим $G^{\prime}=S U(2)$ и $G=S O(3)$. Связности в главных расслоениях $P^{\prime}$ и $P$ описывают соответственно $S U(2)$ - и $S O(3)$-калибровочные поля янг-миллсовского типа [1]. Группа $S U(2)$ является двулистным накрытием группы $S O(3)$. Следовательно, соответствуюшие алгебры Ли изоморфны, $s u(2) \approx s o(3)$. Накрываюший гомоморфизм $f^{\prime}: S U(2) \rightarrow S O(3)$ порождает специальный гомоморфизм расслоений $f: P^{\prime}(M, S U(2)) \rightarrow P(M, S O(3))$. Соответственно сушествует отображение связностей в этих расслоениях, которое фактически описывает трансформацию одной калибровочной модели в другую.

Полезно проследить, как гомоморфизм групn порождает гомоморфизм расслоений. Наиболее простая конструкция главного расслоения $P(M, G)$ реализуется в терминах открытого покрытия $\left\{U_{\alpha}\right\}$ многообразия $M$ и функций перехода. Как известно $[13,1]$, каждое расслоение $P(M, G)$ единственным образом определяется над $M$ заданием открытого покрытия $\left\{U_{\alpha}\right\}$ и набора отображений $\psi_{\beta \alpha}: U_{\alpha} \cap U_{\beta} \rightarrow G$, которые определены в любом непустом пересечении $U_{\alpha} \cap U_{\beta} \subset M$, так что в каждом пересечении $U_{\alpha} \cap U_{\beta} \cap U_{\gamma}$ функции перехода удовлетворяют условию совместности

$$
\psi_{\gamma \alpha}(x)=\psi_{\gamma \beta}(x) \psi_{\gamma \alpha}(x), \quad x \in U_{\alpha} \cap U_{\beta} \cap U_{\gamma} \subset M
$$

Исходя из главного расслоения $P^{\prime}(M, S U(2))$ рассмотрим открытое покрытие $\left\{U_{\alpha}\right\}$ и соответствующие функции перехода $\psi_{\beta \alpha}^{\prime}: U_{\alpha} \cap U_{\beta} \rightarrow S U(2)$. Для того же самого покрытия $M$ определим функции перехода $\psi_{\beta \alpha}: U_{\alpha} \cap U_{\beta} \rightarrow S O(3)$ соотношением

$$
\psi_{\beta \alpha}=f^{\prime} \circ \psi_{\beta \alpha}^{\prime}
$$

Напомним, что гомоморфизм групп $f^{\prime}$ описывается уравнением $\sigma_{b} \Lambda_{a}^{b}=g \sigma_{a} g^{-1}$, где $\Lambda^{b}{ }_{a} \in S O(3), g \in S U(2)$ и $\sigma_{a}$ суть $(2 \times 2)$-матришы Паули. Очевидно, что $f^{\prime}\left(g_{1} g_{2}\right)=$ $f^{\prime}\left(g_{1}\right) f^{\prime}\left(g_{2}\right)$, и мы легко убеждаемся, что условие совместности (2.2) выполнено. Построенные функции перехода $\psi_{\beta \alpha}$ единственным образом определяют главное расслоение $P(M, S O(3))$. Полученный гомоморфизм расслоений порождает связность в $P$ из связности в $P^{\prime}$. В физических терминах данная схема индуцирует $S O(3)$-калибровочный потенциал $\Gamma^{a b}$ из $S U(2)$-калибровочного поля $A^{a}$. Локально имеем, что

$$
\Gamma^{a b}=A^{c} \varepsilon_{c}^{a b}
$$


Описанная конструкция существует на многообразии $M$ любой размерности. Однако размерности 3 и 4 представляют наибольший интерес как с физической точки зрения, так и из-за некоторых специфических свойств $S U(2)-$ и $S O(3)$-калибровочных теорий в данных размерностях.

Рассмотрим теперь геометрию Римана-Картана на многообразии $M$. По определению пространство-время Римана-Картана есть $n$-мерное гладкое многообразие $M$ вместе с метрикой $g=g_{i j} d x^{i} \otimes d x^{j}$, задаваемой полями ортонормированных реперов и кореперов,

$$
e_{\alpha}=e_{\alpha}^{i} \partial_{i}, \quad \vartheta^{\beta}=\vartheta_{j}{ }^{\beta} d x^{j}
$$

и с линейной связностью

$$
\Gamma^{\alpha \beta}=\Gamma_{i}{ }^{\alpha \beta} d x^{i}=-\Gamma^{\beta \alpha} .
$$

Мы используем обозначения работ $[2,11]$. В частности, греческие индексы $\alpha, \beta, \gamma, \ldots=$ $0,1, \ldots, n-1$ нумеруют компоненты неголономных реперов, тогда как латинские индексы $i, j, k, \ldots=0,1, \ldots, n-1$ относятся к мировым (голономным) координатам; $\wedge$ и 」 обозначают внешнее и внутреннее произведения, соответственно. Неголономные индексы поднимаются и опускаются с помощью метрики плоского пространства

$$
g_{\alpha \beta}=e^{i}{ }_{\alpha} e^{j}{ }_{\beta} g_{i j}=\operatorname{diag}(\underbrace{-1, \ldots,-1}_{s}, \underbrace{1, \ldots, 1}_{n-s}) .
$$

Для $s=1$ мы имеем лоренцеву сигнатуру пространства Минковского, для $s=0$ получаем евклидово пространство. Метрика $g_{\alpha \beta}$ определяет оператор дуализации Ходжа на $M$ (обозначаемый в дальнейшем знаком *).

В $n$-мерной пуанкаре-калибровочной теории (случай $n=4$ см., например, в [2]) 1-формы корепера $\vartheta^{\alpha}$ и связности $\Gamma^{\alpha \beta}$ рассматриваются как калибровочные потении$a л b l$, отвечающие локальным трансляциям и локальным лоренцевым преобразованиям, соответственно. Напряженности калибровочных полей даются для транслящии 2-формой кручения

$$
T^{\alpha}:=D \vartheta^{\alpha}=d \vartheta^{\alpha}+\Gamma_{\beta}^{\alpha} \wedge \vartheta^{\beta}=\frac{1}{2} T_{i j}{ }^{\alpha} d x^{i} \wedge d x^{j}
$$

и для локальных лоренцевых преобразований - 2-формой кривизны

$$
R^{\alpha \beta}:=d \Gamma^{\alpha \beta}+\Gamma_{\gamma}^{\beta} \wedge \Gamma^{\alpha \gamma}=\frac{1}{2} R_{i j}^{\alpha \beta} d x^{i} \wedge d x^{j}=-R^{\beta \alpha} .
$$

Форма кривизны антисимметрична по индексам $\alpha$ и $\beta$. В обшем случае кривизна Римана-Картана имеет больше неприводимых компонент, чем риманова кривизна.

При размерности $n \geqslant 3$ кручение и кривизна удовлетворяют тождествам Бианки

$$
D T^{\alpha}-R_{\gamma}^{\alpha} \wedge \vartheta^{\gamma} \equiv 0, \quad D R_{\alpha}^{\beta} \equiv 0
$$

Теория гравитации Эйнштейна-Картана в пространстве-времени любого числа измерений определяется лагранжианом Гильберта-Эйнштейна

$$
L_{\mathrm{HE}}=-\frac{1}{2} \eta_{\alpha \beta} \wedge R^{\alpha \beta}
$$

где $\eta_{\alpha \beta}:={ }^{*}\left(\vartheta_{\alpha} \wedge \vartheta_{\beta}\right)$. Интересно отметить, что при $n=3$ этот лагранжиан описьвает топологическую (так называемую BF) теорию поля. 


\section{3. ТЕОРИЯ ЯНГА-МИЛЛСА В ЧЕТЫРЕХ И ТРЕХ ИЗМЕРЕНИЯХ}

Рассмотрим $S U(2)$-калибровочную теорию на плоском пространстве-времени Минковского $M$. Это четырехмерное многообразие, $\operatorname{dim} M=4$, которое имеет структуру произведения $R \times \underline{M}$ с плоским евклидовым 3-мерным пространством $\underline{M}$. Хотя $M$ и $\underline{M}$ оба являются плоскими и калибровочная теория ковариантна относительно глобальных лоренцевых преобразований декартовых координат на $M$ и $\underline{M}$, ясно, что всегда можно использовать произвольные криволинейные координаты. Действительно, все основные физические объекты являются внешними формами: 1-форма потенциала калибровочного поля $A=A^{a} \tau_{a}$ и 2-форма

$$
F=\left(d A^{a}+\frac{1}{2} \varepsilon_{b c}^{a} A^{b} \wedge A^{c}\right) \tau_{a}
$$

напряженности калибровочного поля. Эти формы не зависят от локальных координат (матрицы $\tau_{a}$ образуют базис алгебры Ли). Будем обозначать произвольные локальные координаты $t$ (или $x^{0}$ ) на $R$ и $x^{i}, i=1,2,3$, на $\underline{M}$. (Псевдо) риманова структура вводится на $M$ метрикой Минковского $g$ с лоренщевой сигнатурой $(-,+,+,+)$. Эта геометрическая структура не зависит от полей Янга-Миллса и трактуется как фиксированный фон. Выберем локальные координаты $\left\{t, x^{i}\right\}$, в которых

$$
g=-d t \otimes d t+\underline{g}=-d t \otimes d t+\underline{g}_{i j} d x^{i} \otimes d x^{j},
$$

где $\underline{g}=\underline{g}_{i j} d x^{i} \otimes d x^{j}$ - положительно-определенная 3-метрика. Последняя является плоской, т.е. сушествует преобразование от $x^{i}$ к координатам $x^{\prime}$, в которых $\underline{g}_{i j}^{\prime}=\delta_{i j}$. Здесь и далее подчеркнутые величины - трехмерные объекты и операторы на $\underline{M}$. В частности, $d$ - полный четырехмерный внешний дифференциал, а 3 -дифференциал на $\underline{M}$ обозначается $\underline{d}$. В локальных координатах имеем $d=d t \partial_{0}+d x^{i} \partial_{i}, \underline{d}=d x^{i} \partial_{i}$.

Предположим, что временная координата $t$ фиксирована. Такое координатное калибровочное условие определяет $(1+3)$-разбиение $M$. Нетрудно построить $(1+3)$-разбиение [5] теории Янга-Миллса на $M$,

$$
A=d t A_{0}+\underline{A}, \quad F=d t \wedge E+B .
$$

Напряженности поля Янга-Миллса разбиваются на "электрическую" и "магнитную" части, определяемые соответственно соотношениями

$$
E:=\underline{A}-\underline{D} A_{0}, \quad B:=\underline{F}=\underline{d} \underline{A}+\underline{A} \wedge \underline{A},
$$

где $\underline{A}=\partial_{0} \underline{A}$ - временная производная потенциала. Инфинитезимальное калибровочное преобразование разбивается на пару преобразований $\delta A_{0}=-D_{t} \xi, \delta \underline{A}=-\underline{D} \xi$, где $\xi=$ $\xi^{a} \tau_{a}$ - параметр бесконечно малого преобразования и $D_{t}:=\partial_{0}+\left[A_{0},\right], \underline{D}:=\underline{d}+[\underline{A}$,$] .$ "Электрическая" и "магнитная" части преобразуются соответственно как $\delta E=[\xi, E]$ и $\delta B=[\xi, B]$.

Лагранжиан Янга-Миллса

$$
L_{\mathrm{YM}}=-\frac{1}{2} F^{a} \wedge^{*} F_{a}
$$


где * обозначает четырехмерную дуализацию Ходжа (определенную метрикой (3.1)), представляется в виде

$$
L_{\mathrm{YM}}=\frac{1}{2} d t \wedge\left(E^{a} \wedge^{*} E_{a}-B^{a} \wedge^{*} B_{a}\right),
$$

где обозначает 3-мерную дуализацию Ходжа, которая задается плоской фоновой 3 -метрикой $\underline{g}$. Уравнения поля, получаюшиеся из этого лагранжиана, имеют вид

$$
\begin{aligned}
& \underline{D}^{*} E=0, \quad \underline{D}^{*} B=-D_{t^{*}} E, \\
& \underline{D} B=0, \quad \underline{D} E=D_{t} B .
\end{aligned}
$$

В гамильтоновой формулировке теории Янга-Миллса "электрическая" трехмерная 1-форма $E$ дуальна импульсу, канонически-сопряженному к $\underline{A}$. Нединамическая компонента $A_{0}$ играет роль лагранжева множителя. Это отражает известный факт наличия связей в теории из-за калибровочной симметрии. Во многих случаях удобно выбрать калибровочное условие $A_{0}=0$ и редуцировать теорию только к динамике канонических переменных $\underline{A}, E$. Такая эффективная 3 -мерная модель может быть проквантована обычным образом [8].

\section{4. ТРЕХМЕРНЫЕ ГЕОМЕТРИИ РИМАНА-КАРТАНА,

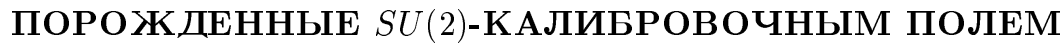

Сушествует несколько отображений трехмерной теории Янга-Миллса в эффективные геометрии Римана-Картана. Они не эквивалентны друг другу, и поэтому имеет смысл рассмотреть все возможные формулировки. Данные отображения описывают построение 1-формы корепера $\theta^{a}$ из 1-формы $S U(2)$-калибровочного поля $A^{a}$ или из соответствуюшей 1-формы $S O(3)$-связности $\Gamma^{a b}$. Заметим, что все эти объекты определены на 3 -мерном многообразии $\underline{M}$ и имеют 9 независимых компонент каждый. Кривизна Римана-Картана и кручение также имеют по 9 компонент в трех измерениях.

4.1. Отображение Лунева. Отображение Лунева [9] определено при наличии фиксированной фоновой геометрии (например, пространства Минковского), что позволяет явно построить действие Янга-Миллса.

Перепишем лагранжиан (3.4) в форме теории первого порядка. Это достигается с помошью двух вспомогательных полей, 1-формы $\Theta_{a}$ и 2-формы $\pi_{a}$ (преобразуюшихся ковариантным образом под действием калибровочной группы):

$$
L_{\mathrm{YM}}=d t \wedge\left(\pi_{a} \wedge E^{a}-\frac{1}{2} \pi_{a} \wedge^{*} \pi^{a}-\Theta_{a} \wedge B^{a}+\frac{1}{2} \Theta_{a} \wedge^{*} \Theta^{a}\right) .
$$

После этого легко установить отображение Лунева следуюшим образом. Для каждой трехмерной янг-миллсовской конфигурации $\left(\underline{A}^{a}, \Theta^{a}\right)$ определим трехмерную әффективную геометрию Римана-Картана соотношениями (сравните с (2.4))

$$
\underline{\Gamma}^{a b}=A^{c} \varepsilon_{c}^{a b}, \quad \underline{\theta}^{a}=\Theta^{a} .
$$


Третий член в лагранжиане (4.1), таким образом, является стандартным лагранжианом Гильберта-Эйнштейна (2.10):

$$
-\Theta_{a} \wedge B^{a}=-\frac{1}{2} \eta_{a b} \wedge \underline{R}^{a b} .
$$

Вьведем эффективные "гравитационные" уравнения поля. Независимая вариация (4.1) относительно $\Gamma^{a b}$ и $\theta^{a}$ приводит соответственно к равенствам

$$
\underline{T}^{a}=-D_{t} \pi^{a}, \quad \underline{R}^{a b}=\varepsilon^{a b c *} \theta_{c} .
$$

Заметим, что в обшем случае эффективная пространственная геометрия действительно является геометрией Римана-Картана с нетривиальными кривизной и кручением. Очевидно, в частности, что кручение в (4.4) не исчезает для нестатических конфигураций. Однако и в статическом случае эффективное кручение отлично от нуля, если $A_{0} \neq 0$. Эффективная метрика (поле кореперов) удовлетворяет уравнениям Эйнштейна (4.4), которые можно переписать в форме

$$
\underline{R}^{a b}=\Lambda \theta^{a} \wedge \theta^{b}+2 \Lambda^{[a}{ }_{c} \theta^{b]} \wedge \theta^{c} .
$$

Здесь тензор

$$
\Lambda^{a b}:=\frac{\sqrt{\operatorname{det} \underline{g}}}{\operatorname{det} \theta} \theta_{i}^{a} \theta_{j}^{b} \underline{g}^{i j}
$$

описывает влияние фиксированной фоновой геометрии пространства-времени на эффективную геометрию, $\Lambda:=\Lambda_{a}^{a}$. В обшем случае вместо пространства-времени Минковского можно рассматривать произвольное искривленное пространство-время.

4.2. Отображение Фридмана. В отличие от отображения Лунева отображение Фридмана [8] определено на пространственно-временном многообразии даже в отсутствие фоновой метрической структуры.

Предположим, что на трехмерном многообразии $M$ задана $S O(3)$-связность $\Gamma_{\alpha}{ }^{\beta}$. Тогда на $M$ можно ввести геометрию Римана-Картана. Описываемая ниже конструкция специфична для размерности 3 и для калибровочной группы $S O(3)$. Заметим, что $M$ изначально не имеет метрики, но специальная риманова структура индуцируется на $M$ связностью $\Gamma_{\alpha}{ }^{\beta}$, если выполнены некоторые необходимые условия. Как мы уже выяснили выше, $S O(3)$-калибровочная теория может рассматриваться как индуцированная, возникающая из $S U(2)$-калибровочной модели. В этом смысле описанная в данном разделе конструкция фактически определяет построение внешнейгеометрии (т.е. обычной геометрии на самом многообразии) из внутренней геометрии расслоенного пространства.

Центральным пунктом оригинального отображения Фридмана является введение неголономного корепера $\vartheta^{\alpha}$ уравнением

$$
R^{\alpha \beta}=\vartheta^{\alpha} \wedge \vartheta^{\beta} .
$$

Левая часть зависит только от связности, и требуется разрешить это уравнение относительно корепера. После этого риманова метрика определяется как

$$
g=g_{\alpha \beta} \vartheta^{\alpha} \otimes \vartheta^{\beta}, \quad g_{i j}=g_{\alpha \beta} \vartheta_{i}^{\alpha} \vartheta_{j}^{\beta} .
$$


Ниже мы дадим формальное не зависяшее от координат решение наряду с явным решением в локальных координатах. В качестве предварительного шага заметим, что уравнение (4.7) имеет 9 компонент, как и число компонент корепера в трех измерениях.

Формальное решение очень простое. Свернув уравнение (4.7) с тензором $\varepsilon_{\alpha \beta \gamma} / 2$ и использовав оператор дуализации Ходжа, определенный метрикой (4.8), найдем

$$
\vartheta^{\gamma}=\frac{1}{2} \varepsilon^{\alpha \beta \gamma \#} R_{\alpha \beta}
$$

Однако имеется одна тонкость. Оператор, отмеченный знаком \# в правой части (4.9), содержит детерминант метрики (4.8), который также следует выразить через компоненты связности. Здесь и далее мы используем обозначение \#, чтобы подчеркнуть отличие этого оператора от обоих операторов Ходжа, помеченных знаками * и *, которые задаются фоновыми метрическими структурами. Описанную проблему проще всего исследовать в локальных координатах.

Вспоминая, что компоненты кривизны определяются из соотношения

$$
\begin{aligned}
R_{\alpha}^{\beta} & =\frac{1}{2} R_{i j \alpha}{ }^{\beta} d x^{i} \wedge d x^{j}= \\
& =\frac{1}{2}\left(\partial_{i} \Gamma_{j \alpha}{ }^{\beta}-\partial_{j} \Gamma_{i \alpha}{ }^{\beta}+\Gamma_{i \gamma}{ }^{\beta} \Gamma_{j \alpha}{ }^{\gamma}-\Gamma_{j \gamma}{ }^{\beta} \Gamma_{i \alpha}{ }^{\gamma}\right) d x^{i} \wedge d x^{j},
\end{aligned}
$$

получим из (4.7) с помошью внешнего произведения с $\vartheta^{\gamma}$ формулу

$$
R^{\alpha \beta} \wedge \vartheta^{\gamma}=\frac{1}{2} R_{i j}^{\alpha \beta} \vartheta_{k}^{\gamma} d x^{i} \wedge d x^{j} \wedge d x^{k}=\vartheta^{\alpha} \wedge \vartheta^{\beta} \wedge \vartheta^{\gamma}=\varepsilon^{\alpha \beta \gamma} \eta
$$

где 3 -форма объема есть $\eta:=\# 1=\operatorname{det} \vartheta d^{3} x$. Используя тождество $d x^{i} \wedge d x^{j} \wedge d x^{k}=$ $\varepsilon^{i j k} d^{3} x$, найдем, что

$$
\frac{1}{4} \varepsilon_{\alpha \beta \mu} \varepsilon^{i j k} R_{i j}^{\alpha \beta} \vartheta_{k}^{\nu}=\delta_{\mu}^{\nu} \operatorname{det} \vartheta
$$

Данное уравнение имеет смысл, только если зависящая от связности $(3 \times 3)$-матрица

$$
B_{\mu}^{k}:=\frac{1}{4} \varepsilon_{\alpha \beta \mu} \varepsilon^{i j k} R_{i j}^{\alpha \beta}
$$

не вырождена. Тогда соотношение (4.12) дает $\operatorname{det} B=(\operatorname{det} \vartheta)^{2}$, и окончательно получаем компоненты репера в виде

$$
e_{\mu}^{k}=\frac{B_{\mu}^{k}}{\sqrt{\operatorname{det} B}} .
$$

Компоненты корепера $\vartheta_{i}^{\alpha}$ составляют обратную матрищу.

Таким образом, необходимым условием существования данной структуры РиманаКартана является невырожденность матрицы (4.13). Заметим, что в обеих частях равенства (4.12) присутствует нумерический объект $\varepsilon$, однако все соотношение целиком является общекоординатно-ковариантным.

Интересно отметить, что уравнения поля в подходе Лунева (4.5) сводятся к фундаментальному соотношению (4.7) в подходе Фридмана, если $\Lambda^{\alpha \beta}=\delta^{\alpha \beta}$. Как вытекает из определения (4.6), это означает, что действительно на $M$ не существует иной независимой метрической структуры, кроме индуцированной геометрии $\underline{g}_{i j}=g_{i j}$. 
Рассмотрим основные свойства геометрии Римана-Картана, определяемой уравнением (4.7). Имея решение для корепера, получаем пуанкаре-калибровочные поля $\left(\vartheta^{\alpha}, \Gamma_{\beta}^{\alpha}\right)$, соответствуюшие 2-формы напряженностей суть исходная $S O(3)$-кривизна и кручение $T^{\alpha}=D \vartheta^{\alpha}$. По построению репера кривизна Римана-Картана постоянна, что выражает соотношение (4.7). В терминах компонент кривизны

$$
R^{\alpha \beta}=\frac{1}{2} R_{\mu \nu}^{\alpha \beta} \vartheta^{\mu} \wedge \vartheta^{\nu}
$$

уравнение (4.7) дает уравнение

$$
R_{\mu \nu}^{\alpha \beta}=\delta_{\mu}^{\alpha} \delta_{\nu}^{\beta}-\delta_{\nu}^{\alpha} \delta_{\mu}^{\beta}
$$

так что тензор Риччи имеет вид $A_{\beta}{ }^{\alpha}:=R_{\beta \nu}{ }^{\alpha \nu}=2 \delta_{\beta}^{\alpha}$ и скаляр кривизны равен $A:=$ $A_{\alpha}{ }^{\alpha}=6$.

Обратимся теперь к свойствам кручения. Подставив уравнение (4.7) в тождества Бианки (2.9), найдем

$$
\left.T^{\alpha} \wedge \vartheta^{\beta}-\vartheta^{\alpha} \wedge T^{\beta}=0 \longrightarrow T:=e_{\alpha}\right\rfloor T^{\alpha}=0
$$

и

$$
D T^{\alpha}=0
$$

Уравнение (4.16) показывает, что след кручения равен нулю, и остаются только $5 \oplus 1$ независимых компонент кручения.

Если разложить 1-форму дуального кручения относительно корепера, ${ }^{2} T^{\alpha}=$ $\widetilde{T}_{\beta}^{\alpha} \vartheta^{\beta}$, то получим обшее представление кручения в $3 D$-пространстве:

$$
T^{\alpha}=\frac{1}{2} \widetilde{T}^{\alpha \beta} \varepsilon_{\beta \gamma \delta} \vartheta^{\gamma} \wedge \vartheta^{\delta}
$$

Подстановка в уравнение (4.16) приводит к условию

$$
\widetilde{T}_{\alpha \beta}=\widetilde{T}_{\beta \alpha},
$$

тогда как подстановка (4.18) в (4.17) дает дифференциальное условие на матрицу коэффициентов:

$$
D_{\beta} \widetilde{T}^{\alpha \beta}=0 .
$$

Рассмотренная конструкция была предложена в работах [8] с явным использованием локальных координат.

4.3. Новое отображение Джонсона. Отображение Фридмана не является взаимно однозначным. А именно, возможна ситуация, при которой различные янг-миллсовские поля $\Gamma^{a b}$ отображаются в одну и ту же 1 -форму корепера $\theta^{a}$. Это связано с известной проблемой неоднозначности Ву-Янга $[14,15]$, которая обсуждается в разделе 5. Чтобы исключить данную проблему, в работе [12] было предложено отображение,

4 Теоретическая и математическая физика, т. 117, № 2, 1998 г. 
которое мы далее будем называть “новым отображением Джонсона". В этой работе корепер $\theta^{a}$ строится таким образом, что 1-форма связности $\Gamma^{a b}$ является чисто римановой (связность Леви-Чивиты) связностью без кручения. Математически такой корепер определяется дифференциальным уравнением

$$
d \theta^{a}+\Gamma_{b}^{a} \wedge \theta^{b}=0
$$

Важно отметить, что новое отображение Джонсона существует только в трех измерениях, где число уравнений (4.21) равно 9, т.е. равно числу неизвестных компонент.

Ясно, что новое отображение Джонсона также не является взаимно однозначным: одной и той же связности $\Gamma^{a b}$ может отвечать несколько разных кореперов $\theta^{a}$ (простейшим примером являются 1-формы $\theta^{a}$ и $C \theta^{a}$ с константой $C$, которые обе удовлетворяют уравнению (4.21)). Более сложный пример неоднозначности нового отображения Джонсона рассмотрен в разделе 5 .

Кроме решения проблемы неоднозначности Ву-Янга, отображение (4.21), по-видимому, устанавливает корректное описание конструкции Голдстоуна-Джакива [16] для квантования теории Янга-Миллса в терминах калибровочно-инвариантных полевых переменных.

4.4. Метрическая структура в формализме расслоений. Дадим глобальную формулировку теории отображений на языке расслоенных пространств. Для конкретности рассмотрим отображение Фридмана.

Основным моментом является введение метрики на многообразии $M$. На заданном гладком многообразии $M$ фундаментальную роль играет главное расслоение линейных реперов $L(M)$. Напомним, что линейный репер в точке $x \in M$ есть произвольный базис $\left\{e_{\alpha}\right\}$ (в нашем случае $\alpha=1,2,3$ ) касательного пространства $T_{x} M$. Множество всех реперов над всеми точками составляет многообразие $L(M)$ (гладкая структура легко вводится). Произвольная точка $u=\left(x, e_{\alpha}\right) \in L(M)$ отображается в точку $x \in M$ с помошю канонической проекции $\pi: L(M) \rightarrow M$. Структурная группа $L(M)$ есть $G=G L(3, R)$ с очевидным правым действием $g \in G: u \rightarrow R_{g} u=u g=\left(x,(e g)_{\alpha}\right)$ (более подробно, $(e g)_{\alpha}=e_{\beta} g_{\alpha}^{\beta}$ с $(3 \times 3)$-матрицей $\left.g_{\alpha}^{\beta} \in G L(3)\right)$.

Задание метрики на $M$ тесно связано с редукцией расслоения линейных реперов $L(M)$ к подрасслоению так называемых ортонормированных реперов $O(M)$. Для группы врашений $H=O(3, R)$, рассматриваемой как подгруппа $H \subset G$, определено очевидное правое действие на $L(M), h \in H: u \rightarrow R_{g} u=u g=\left(x,(e h)_{\alpha}\right)$, которое вводит фактор-пространство $E=L(M) / H$. Обозначим "начало отсчета" в фактор-группе $G / H$ как $\xi_{0}$ (т.е. это класс смежности $H$, задаваемый единичным элементом группы $G$ ). Тогда произвольную точку $v \in E$ можно построить как $v=\left(x, e \xi_{0}\right)$. Легко видеть [13], что $E$ является векторным расслоением, ассоциированным с $L(M)$, которое обладает структурной группой $G$ и стандартным слоем $G / H$. В свою очередь, расслоение линейных реперов $L(M)$ можно рассматривать как главное расслоение $P(E, H)$ над базой $E$ со структурной группой $H$. Каноническая проекция $\mu: L(M) \rightarrow E$ задается с помошью факторизации проекции $G \rightarrow G / H$. Векторное расслоение $E$ есть источник метрических структур на $M$

Действительно, локальные сечения $E$ (т.е. такие отображения $\sigma: M \rightarrow E$, что $\left.\pi_{E} \circ \sigma=\mathrm{id}\right)$ находятся во взаимно однозначном соответствии с редукциями $L(M) \mathrm{k}$ 
$O(M)$, а следовательно, и с римановыми метриками на $M$. Покажем это. Пусть задано сечение $\sigma: M \rightarrow E$. Тогда подрасслоение ортонормированных реперов определим как множество

$$
O(M):=\{u \in L(M) \mid \mu(u)=\sigma(\pi(u))\},
$$

т.е. отображение $\sigma$ в каждой точке $M$ выбирает один из классов эквивалентности, на которые множество реперов $e_{\alpha}$ разбито действием группы врашений. Более подробно можно записать, что $\sigma(x)=v(x)=\left(x, g(x) \xi_{0}\right)$, т.е. для точки $u=\left(x, e_{\alpha}\right)$ условие (4.22) означает, что $e \xi_{0}=g(x) \xi_{0}$. Тем самым все реперы над точкой $x \in M$, составляюшие слой $O(M)$ в $x$, связаны с представителем $g(x)$ преобразованием из подгруппы $H=O(3, R)$. Мы отсылаем читателя к книге [13] за строгим доказательством того, что $O(M)$ есть гладкое многообразие, вложенное в $L(M)$. Обратное также верно. Если задано отображение редукции $f: O(M) \rightarrow L(M)$, сечение $E$ строим следуюшим образом: $\sigma(x)=\mu(f(u))$ для $x \in M$, где $u \in O(M)$ - произвольный элемент над $x$ (т.е. $\pi(u)=x)$. Это определение корректно, поскольку для правого действия $h \in H$ имеем $f(u h)=f(u) h$, и, следовательно, $\mu(f(u h))=\mu(f(u))$.

Каждая редукция $L(M)$ к $O(M)$ эквивалентна введению метрики на $M$ (и этот факт объясняет, в частности, почему $O(M)$ называется расслоением ортонормированныц реперов - относительно чего они "ортонормированы"). Покажем это следующим образом [13]. В $R^{3}$ существует естественное внутреннее произведение (,), которое в стандартном базисе $E_{\alpha}$ в $R^{3}$ задается как $\left(E_{\alpha}, E_{\beta}\right)=\delta_{\alpha \beta}$ (напомним, что $E_{1}=(1,0,0)$, $\left.E_{2}=(0,1,0), E_{3}=(0,0,1)\right)$ и, очевидно, является $O(3, R)$-инвариантным. Тогда для редуцированного расслоения $O(M)$ риманову метрику $g$ на $M$ определим для любых касательных векторов $X, Y \in T_{x} M$ равенством

$$
g(X, Y):=\left(u^{-1} X, u^{-1} Y\right)
$$

где $u \in O(M)$ - произвольный элемент над $x \in M$, рассматриваемый как линейный изоморфизм $R^{3}$ на касательное пространство. Данное определение не зависит от выбора $u$, поскольку произведение (,) является $O(3, R)$-инвариантным.

Как мы видели, метрика на $M$ однозначно определена сечением векторного расслоения $E=L(M) / O(3)$. Собственно сечение может быть задано с помошью различных конкретных конструкций.

Покажем, что геометрия Римана-Картана, индуцированная на $M$ связностью на главном $S O(3)$-расслоении, определена корректно и в полном согласии с общей схемой в терминах расслоенных пространств и редукций. Доказательство следует непосредственно. Связность Г задает с помошью (4.7) локальное сечение расслоения линейных реперов $L(M)$. Это сечение является полем реперов с компонентами (4.14). Обозначим данное сечение как $\sigma_{\Gamma}: M \rightarrow L(M)$. Тогда требуемое сечение фактор-расслоения $E$, очевидно, определяется как $\sigma(x)=\mu\left(\sigma_{\Gamma}\right)$. Такое определение совместно с калибровочной $S O(3)$-свободой исходной внутренней калибровочной теории: при преобразовании связности $\Gamma^{\prime}{ }_{\alpha}^{\beta}=\Lambda_{\delta}^{-1 \beta} \Gamma_{\gamma}{ }^{\delta} \Lambda_{\alpha}{ }^{\gamma}+\Lambda_{\gamma}^{-1 \beta} d \Lambda_{\alpha}{ }^{\gamma}$ с $\Lambda_{\beta}{ }^{\alpha}(x) \in S O(3)$ сечение расслоения реперов переходит с учетом (4.7), (4.14) в другое сечение. Последнее, однако, связано просто врашением с первым сечением, $e_{\alpha}^{\prime}=\Lambda_{\alpha}^{\beta} e_{\beta}$, и, следовательно, $\mu\left(\sigma_{\Gamma^{\prime}}\right)=\mu\left(\sigma_{\Gamma}\right)$. 


\section{5. ЭФФЕКТИВНОЕ КРУЧЕНИЕ И НЕОДНОЗНАЧНОСТИ}

Эффективная геометрия Римана-Картана дает калибровочно-инвариантное описание конфигураций полей Янга-Миллса. Это делает формализм отображений полезным для исследования проблемы калибровочной эквивалентности полей. Как уже отмечалось, отображение Фридмана чувствительно к неоднозначности Ву-Янга.

Первый известный пример неоднозначности ("копий") калибровочных полей, который дается в работе [14], приводит к вырожденной эффективной геометрии РиманаКартана, поскольку тогда $\operatorname{det} B=\operatorname{det} \vartheta=0$ в уравнениях (4.7), (4.13). Неединственное решение уравнения (4.7) в этом случае описывает геометрию двумерной сферы вместо регулярного трехмерного многообразия. Вырожденность геометрии в примере Ву-Янга легко понять, если заметить, что данные конфигурации калибровочных полей являются эффективно двумерными. Действительно, прямая проверка показьвает, что потенциалы Ву-Янга, записанные в сферических координатах $(r, \theta, \phi)$, раскладываются только по диффференциалам угловых координат $d \theta, d \phi$, а коэффициенты этих разложений суть функции только $\theta$ и $\phi$ (и не зависят от $r$ ). Таким образом, можно говорить о том, что эти калибровочные поля “живут” на двумерной сфере, вложенной в трехмерное пространство.

Более интересно найти нетривиальный трехмерный пример неоднозначности ВуЯнга. Недавняя работа [17] в этом направлении частично некорректна, однако ниже мы приведем пример с аналогичным анзацем. В отличие от [17], мы не станем решать уравнение (4.7) относительно кручения. Вместо этого мы используем формализм первого порядка и рассмотрим задачу в терминах индуцированного корепера $\vartheta^{\alpha}$ и $S O(3)$-связности $\Gamma_{\beta}{ }^{\alpha}$. Основной анзац имеет вид

$$
\Gamma_{\alpha}{ }^{\beta}=\left(\begin{array}{ccc}
0 & k \vartheta^{3} & \varphi_{1} \\
-k \vartheta^{3} & 0 & \varphi_{2} \\
-\varphi_{1} & -\varphi_{2} & 0
\end{array}\right)
$$

где $k=$ const и 1-формы $\varphi_{1}, \varphi_{2}$ таковы, что $\left.\left.e_{3}\right\rfloor \varphi_{1}=e_{3}\right\rfloor \varphi_{2}=0$. Если сушествуют локальные координаты $(x, y, z)$, в которых $\vartheta^{1}=d x, \vartheta^{2}=d y, \vartheta^{3}=d z$, то можно выписать явное решение уравнения (4.7) в форме

$$
\begin{aligned}
& \varphi_{1}=\sqrt{k^{-2}-1}\left(\cos (k z) \vartheta^{1}+\sin (k z) \vartheta^{2}\right)-\frac{1}{k} \vartheta^{2}, \\
& \varphi_{2}=\sqrt{k^{-2}-1}\left(\sin (k z) \vartheta^{1}-\cos (k z) \vartheta^{2}\right)+\frac{1}{k} \vartheta^{1} .
\end{aligned}
$$

Расчет дает 2-форму кручения для данных кореперов $\vartheta^{\alpha}$ и связностей $(5.1),(5.2),(5.3)$ в виде

$$
T^{1}=\vartheta^{3} \wedge\left(k \vartheta^{2}-\varphi_{1}\right), \quad T^{2}=-\vartheta^{3} \wedge\left(k \vartheta^{1}+\varphi_{2}\right), \quad T^{3}=-\frac{2}{k} \vartheta^{1} \wedge \vartheta^{2} .
$$

Наконец, остается отметить, что $S U(2)$-связность, которая индуцирует данную геометрическую структуру Римана-Картана, описывается калибровочными потенциалами

$$
A^{1}=-\varphi_{2}, \quad A^{2}=\varphi_{1}, \quad A^{3}=-k \vartheta^{3} .
$$


Легко проверить, что 2-форма напряженности Янга-Миллса для потенциалов (5.5) имеет вид

$$
F^{\alpha}=\frac{1}{2} \varepsilon^{\alpha}{ }_{\beta \gamma} \vartheta^{\beta} \wedge \vartheta^{\gamma} .
$$

Для любых значений параметра $k$ калибровочные поля (5.5) имеют одну и ту же 2-форму напряженности (5.6), и, таким образом, индуцированная метрика одинакова. Однако соответствуюшие 2-формы кручения различны, и, следовательно, не сушествует калибровочного преобразования, с помошью которого можно перевести конфигурации из семейства (5.5) друг в друга. Более того, инвариант кривизны явно зависит от параметра $k,{ }^{\#} T^{\alpha} \wedge T_{\alpha}=2 \eta\left(4 k^{-2}+1+k^{2}\right)$. Это означает не только калибровочную неэквивалентность относительно внутренних преобразований $S U(2) \approx S O(3)$, но также невозможность построить преобразование координат, которое связывало бы элементы семейства (5.5). Мы имеем, таким образом, явньй пример трехмерной невырожденной неоднозначности $B y-Я_{\text {нг }}$ в определении напряженности калибровочного поля.

В подходе, основанном на "новом отображении Джонсона", эффективное кручение равно нулю по определению, и тем самым проблемы неоднозначности Ву-Янга не возникает. Однако нетрудно видеть, что появляется новая неоднозначность. Проиллюстрируем это на примере классической конфигурации Ву-Янга [14], когда $A^{a}=$ $(\Phi-1) r^{-2} \varepsilon^{a}{ }_{i j} x^{i} d x^{j}$, где $\Phi=$ const и $x^{i}, i=1,2,3$, суть декартовы координаты с $r^{2}:=x^{i} x_{i}$. Решение уравнения (4.21), задаюшего новое отображение Джонсона, описывается широким семейством невырожденных кореперов

где

$$
\vartheta^{a}=f(r) d x^{a}+h(r) \frac{x^{a} x_{b}}{r^{2}} d x^{b},
$$

$$
f=r^{\Phi-1}\left(C+\int^{r} \frac{h(\tilde{r})}{\tilde{r}^{\Phi}} d \tilde{r}\right)
$$

с константой интегрирования $C$ и произвольной функиией $h(r)$.

\section{6. ЗАКЛЮЧИТЕЛЬНЫЕ ЗАМЕЧАНИЯ}

Установление прямых соответствий между геометрическими структурами калибровочной теории Янга-Миллса и теории гравитации полезно и поучительно во многих отношениях как в классической, так и в квантовой областях. Явное устранение нединамических степеней свободы и формулировка квантовой калибровочной теории в терминах калибровочно-инвариантных переменных обсуждались ранее в работах $[8,9,12]$. Исследование соответствий меж ду классическими решениями проливает новый свет на физическое содержание как "внутренних", так и "внешних" калибровочных моделей. По-видимому, Виттен [18] первым заметил связь между самодуальными конфигурациями $S U(2)$-калибровочных полей и решениями эйнштейновской обшей теории относительности. Недавно внимание к данному вопросу привлекли результаты Лунева [9] и Синглтона [19], которые получили новые сферически-симметричные решения уравнений Янга-Миллса с запирающими свойствами исходя из решений типа Шваршшильда трехмерной теории гравитации. Аналогичные аксиально-симметричные решения ("черные струны”) обсуждаются в работах [20].

Данная работа выполнена в рамках проекта Не 528/17-2, финансировавшегося Deutche Forschungsgemeinschaft (Бонн). 


\section{Список литературы}

[1] А.А. Славнов, Л.Д. Фаддеев. Введение в квантовую теорию калибровочных полей. М.: Наука, 1978; M. Daniel, C. M. Viallet. Rev. Mod. Phys. 1980. V. 52. P. 175.

[2] F. W. Hehl, P. von der Heyde, G.D. Kerlick, J. M. Nester. Rev. Mod. Phys. 1976. V. 48. Р. $393 ;$ В. Н. Пономарев, А. О. Барвинский, Ю. Н. Обухов. Геометродинамические методы и калибровочный подход к теории гравитационных взаимодействий. М.: Энергоатомиздат, 1985; E. W. Mielke. Geometrodynamics of Gauge Fields: On the geometry of Yang-Mills and gravitational gauge theories. Berlin: Akademie-Verlag, 1987; F. W. Hehl, J.D. McCrea, E. W. Mielke, Y. Ne'eman. Phys. Rep. 1995. V. 258. P. 1.

[3] T. D. Lee. Particle physics and introduction to field theory, Contemporary Concepts in Physics. V.1. Revised and updated edition. Chur: Harwood Academic Publ., 1990.

[4] A. Ashtekar. Lectures on non-perturbative canonical gravity. Advanced Ser. in Astrophysics and Cosmology. V. 6. Singapore: World Scientific, 1991.

[5] B. P. Dolan. Phys. Lett. B. 1990. V. 233. P. 89; R. P. Wallner. Phys. Rev. D. 1990. V. 42. P. 441; E. W. Mielke. Ann. Phys. (USA). 1992. V. 219. P. 78.

[6] J. M. Nester, R.-S. Tung, Y.Z. Zhang. Class. Quantum Gravit. 1994. V. 11. P. 757.

[7] E. V. Shuryak. The QCD vacuum, hadrons and the superdense matter, World Scientific Lecture Notes in Physics. V. 8. Singapore: World Scientific, 1988.

[8] K. Johnson. The Yang-Mills ground state. In: QCD - 20 Years Later, Aachen 1992. Eds P. M. Zerwas, H. A. Kastrup. V. 2. Singapore: World Scientific, 1993. P. 795; D. Z. Freedman, P. E. Haagensen, K. Johnson, J. I. Latorre. The hidden spatial geometry of non-Abelian gauge theories. Preprint CERN-TH.7010/93; M. Bauer, D. Z. Freedman, P. E. Haagensen. Nucl. Phys. B. 1994. V. 428. P. 147; D. Z. Freedman. Spatial geometry of Hamiltonian gauge theories. Preprint CERN-TH.7391/94 (Aug 1994); hep-th/9408052; M. Bauer, D. Z. Freedman. Nucl. Phys. B. 1995. V. 450. P. 209.

[9] Ф. А. Лунев. ЯФ. 1993. Т. 56. Вып.11. С. 238; F. A. Lunev. Phys. Lett. B. 1992. V. 295. P. 99; Ф.А. Лунев. ТМФ. 1993. Т. 94. С. 66; F. A. Lunev. Phys. Lett. B. 1993. V. 311. P. 273; V. 314. P. 21; Mod. Phys. Lett. A. 1994. V. 9. P. 2281; J. Math. Phys. 1996. V. 37. P. 5351.

[10] Y. Ne'eman, Dj. Šijački. Chromogravity: QCD-induced diffeomorphisms. Preprint Tel Aviv University, 1993; $D j$. Šijački. QCD originated dynamical symmetry for hadrons. Preprint hep-th 9311033, November 1993; V. Radovanovic, Dj. Šijački. Class. Quantum Gravit. 1995. V. 12. P. 1791.

[11] E. M. Mielke, Yu. N. Obukhov, F. W. Hehl. Phys. Lett. A. 1994. V. 192. P. 153.

[12] P. E. Haagensen, K. Johnson. Nucl. Phys. B. 1995. V. 439. P. 597.

[13] ШІ. Кобаяси, К. Номидзу. Основы дифференциальной геометрии. Т. 1, 2. М.: Наука, 1981.

[14] T. T. Wu, C. N. Yang. Phys. Rev. D. 1975. V. 12. P. 3843.

[15] S. Deser, W. Drechsler. Phys. Lett. B. 1979. V. 86. P. 189.

[16] J. Goldstone, R. Jackiw. Phys. Lett. B. 1978. V. 74. P. 81.

[17] D. Z. Freedman, R. R. Khuri. Phys. Lett. B. 1994. V. 329. P. 263.

[18] L. Witten. Phys. Rev. D. 1979. V. 19. P. 718.

[19] D. Singleton. Phys. Rev. D. 1995. V. 51. P. 5911

[20] Yu. N. Obukhov. Analogue of black string in the Yang-Mills gauge theory. Preprint Univ. Cologne, August 1996; hep-th/9608011; D. Singleton. J. Math. Phys. 1996. V. 37. P. 4574; Nouvo Cimento A. 1996. V. 109. P. 169. 